\title{
Development And Test Of A Planar R-band Accelerating Structure*
}

\author{
R. Merte, H. Henke, M. Peikert \\ Technische Universität Berlin, EN-2, Einsteinufer 17, D-10587 Berlin, Germany \\ D.Yu \\ Duly Research Inc., CA, USA
}

\begin{abstract}
Planar accelerating structures, so called muffin tins, are of great interest for new accelerating techniques which are operating at high frequencies. At present the upper frequency limit for high power sources is $29.9855 \mathrm{GHz}$ available at CERN. Therefore a new design of a planar traveling wave constant impedance accelerating structure is presented. A fully engineered 37-cell prototype with an operating frequency of $29,9855 \mathrm{GHz}$, which is designed for the $2 \pi / 3$ mode, was fabricated by $\mathrm{CNC}$ milling technology. The design includes a power coupler, a cavity geometry optimized to compensate the effect of transverse forces, vacuum flanges and beam pipe flanges. Shown are the frequency scan of transmission and reflection measurements compared to numerical simulations with GdfidL [1]. Further, a non resonant bead pull measurement was made to determine and verify the fundamental modes of the structure. The cavity is planned to be powered at the CLIC test stand at CERN.
\end{abstract}

\section{INTRODUCTION}

Planar structures are very different as compared to round structures. The accelerating mode excites transverse forces which depend on the longitudinal and transverse position. The excitation of higher order modes is expected to be much weaker in the vertical plane, and the surface quality and the shaping of the irises are different. This will affect the power handling compatibility and the beam dynamics. Therefore and because planar structures have never been used to accelerate particles, it was decided to build a fully engineered cavity and to test it under real conditions at the highest possible frequency.

In a collaboration with the CLIC team at CERN it is planned to make the experiments at the CLIC test facility this summer. If possible two experiments are planned. First, the planar structure shall be powered by the drive beam and generate RF power in the multi MW regime. In a second experiment the planar structure shall be fed from the CLIC transfer structure and powered by a test beam.

The planar structure is a 37-cell, $2 \pi / 3$-mode traveling muffin-tin operating at $29.9855 \mathrm{GHz}$. The depth and width of the cavities were chosen such that the transverse forces have equal magnitude while the sign is opposite. Such a

\footnotetext{
${ }^{*}$ Work supported by DOE SBIR grant DE-FG03-96ER82213
}

design allows for the compensation of the forces by rotating every other structure by $90^{\circ}$ around the longitudinal axis, resulting in a weak focusing effect.

The design of the structure [2], the machining of a model scaled up to $10 \mathrm{GHz}$ and measurements at $10 \mathrm{GHz}$ [4] as well as $29.9855 \mathrm{GHz}$, were performed at the TU-Berlin. Two prototypes were machined by industry, the flanges provided by CERN. One prototype was brazed at the HMI, Berlin, the other will be brazed at CERN.

\section{GEOMETRY OF THE STRUCTURE AND RF PARAMETERS}

The structure is a traveling wave, constant impedance structure at $29.986 \mathrm{GHz}$ [2]. From the wavelength of $\lambda=9.997$ $\mathrm{mm}$ follows a period length of $p=3.332 \mathrm{~mm}$ for the $2 \pi / 3$-mode. The iris thickness has been fixed to $t=0.7$ $\mathrm{mm}$. The length of one cavity in beam direction, the gap, results therefore to $g=p-t=2.632 \mathrm{~mm}$. The aperture $2 a$, the width $w$ and the depth $2 b$ of the cavity are the parameters which adjust the mentioned $F_{x}=F_{y}$ property and the frequency. The aperture for the beam influences bandwidth, shunt impedance, wakefields and transverse forces. Thus a parameter analysis of these general cavity dimensions was necessary. The best results have been found for an aperture to wavelength ratio of $a / \lambda=0.18$, resulting in $2 a=3.6 \mathrm{~mm}$. The frequency was adjusted by the cavity width and depth of $w=6.688 \mathrm{~mm}$ and $2 b=8.4 \mathrm{~mm}$, respectively. Figure 1 shows a planar cavity in different views.

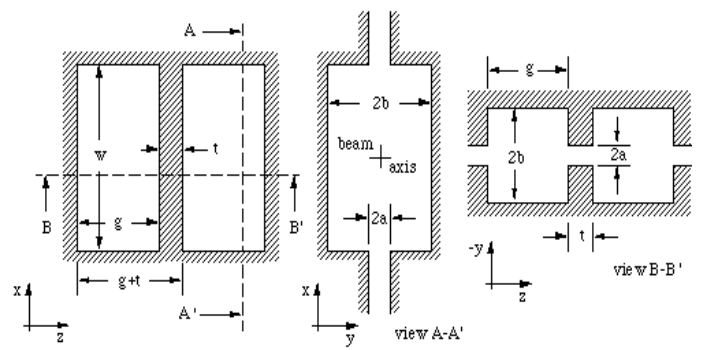

Figure 1. Typical cavity, a) top view, b) cross section and c) longitudinal cut.

The basic RF parameters are determined numerically with GdfidL [1] and listed in table 1. For the determination of the maximum length $L_{\max }$ of the structure we used an attenuation parameter of $\tau=\alpha l=0.8$, resulting in a 
maximal number of cells of $N_{\max }=375$. A test model should have a small number of cells, but it should be large enough to present a measurable impedance. We chose 37 cells for the prototype.

$$
\begin{gathered}
r / Q_{0}=22,62 \mathrm{k} \Omega / \mathrm{m} \\
Q_{0}=4420, r=100 \mathrm{M} \Omega / \mathrm{m} \\
v_{g} / c_{0}=11,2 \% \\
\alpha=0,64 \mathrm{~m}^{-1} \\
\rightarrow \tau=0.8 \Rightarrow L_{\max }=1,25 \mathrm{~m} \Rightarrow N_{\max }=375
\end{gathered}
$$

Table 1. RF parameters of a $F_{x}=F_{y}$ cavity.

\section{MECHANICAL DESIGN}

A 37-cell accelerating section has been modeled, consisting of 35 accelerating cells with $F_{x}=F_{y}$ property and terminated with two coupling cells. The input and output couplers are matched with a cut iris. The power couplers are planar and the input and output ports are from one side. The matching procedure has been done in the time domain with GdfidL [1].

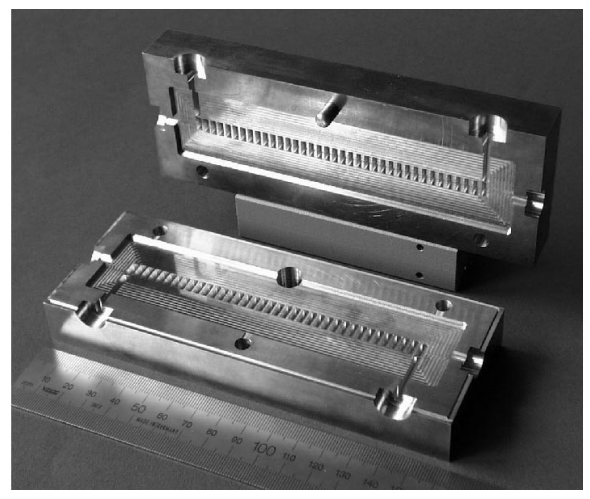

Figure 2. Manufactured 37-cell structure.

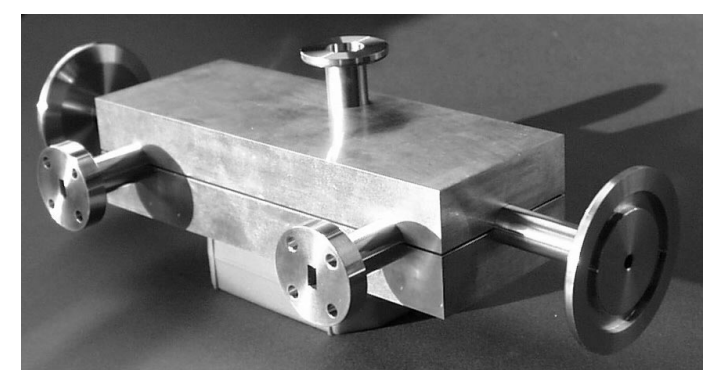

Figure 3. Manufactured structure with RF-, beam pipe- and vacuum-flanges.

The structure was produced by CNC milling technology with an accuracy of $0.004 \mathrm{~mm}$. The machining by inline milling has the disadvantage that cavity corners are rounded with the radius of the milling cutter. The rounded corners cause a frequency shift and detune the match, as recently seen in measurements of X-band models [3], [4]. In order to take account of these effects all simulations are made with rounded corners.

The structure is fully engineered for a high power experiment in vacuum. The design includes ports for vacuum, RF and beam pipe. Figure 2 shows a picture of the manufactured structure and figure 3 the mounted structure with RF-, beam pipe- and vacuum-flanges, ready for brazing. Thermal control for cooling and tuning is also planned. Cooling tubes will be glued on the top and the bottom of the structure.

\section{MEASUREMENT}

The main interest was to see how the $S_{11}$ and $S_{21}$ parameters of the numerical simulation compare to the measurement, as well as in the field patterns of the fundamental modes.

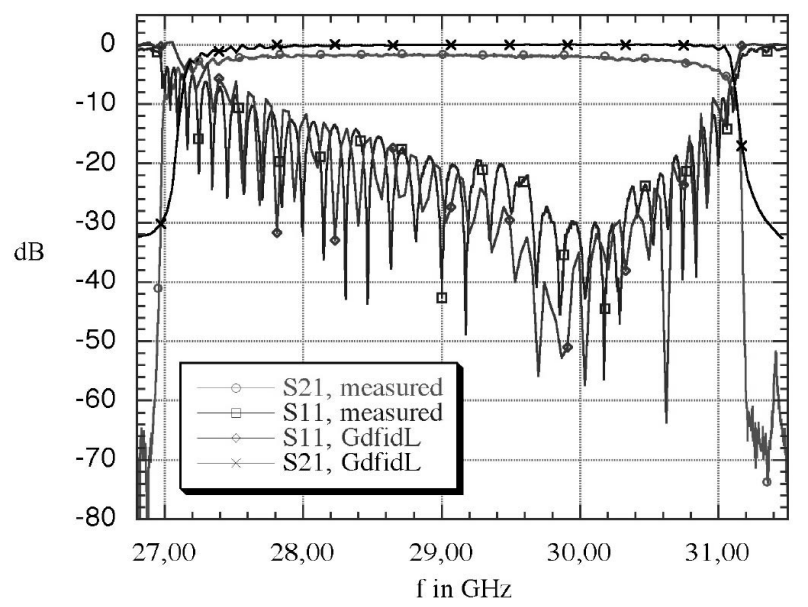

Figure 4. Comparison of the unperturbed measurement with the GdfidL simulation.

\subsection{Frequency scan}

The measurements were made with a Hewlett Packard HP$8722 \mathrm{C}$ vector network analyzer (NWA), working in the frequency range from $50 \mathrm{MHz}$ up to $40 \mathrm{GHz}$. The first step was a full 2-port calibration in the frequency range from $28.8 \mathrm{GHz}$ up to $31.5 \mathrm{GHz}$ using a HP-11644A waveguide calibration kit. A second measurement was made with the fiber inside the structure, but no bead. The fiber is a perturbation by itself and causes a frequency shift. Table 2 gives an overview of the measured frequencies compared to the data obtained with GdfidL [1]. The relation between the $S_{11}$ chart and the dispersion chart is given by:

$$
\#-\text { mode }=\frac{\pi(\mathrm{i}-1)}{\mathrm{N}-1},
$$

where $\mathrm{N}$ is the number of cells (37) and $\mathrm{i}$ is the number of the peak $\mathrm{i}=1 \ldots 37$.

The mentioned rounded corners lead to the further problem that the numerical simulation needs a huge amount 
of RAM and time. Therefore the 37-cell GdfidL simulation was terminated before the steady state was reached. Figure 4 shows a comparison of the $S_{11}$ and $S_{21}$ parameters of the measured unperturbed 37-cell structure with the terminated simulation. You can see a good quantitative agreement concerning the bandwidth and the match for the $2 \pi / 3$-mode, but also unexpected high losses in transmission.

$\begin{array}{ccccc}\begin{array}{c}\text { peak } \\ \text { NO }\end{array} & \begin{array}{c}\text { target } \\ \text { mode }\end{array} & \begin{array}{c}\text { unperturbed } \\ f_{u p}[\mathbf{G H z}]\end{array} & \begin{array}{c}\text { perturbed } \\ f_{p}[\mathbf{G H z}]\end{array} & \begin{array}{c}\text { GdfidL } \\ f[\mathbf{G H z}]\end{array} \\ 13 & \pi / 3 & 28.302 & 28.086 & - \\ 19 & \pi / 2 & 29.162 & 28.954 & - \\ 25 & 2 \pi / 3 & 30.012 & 29.985 & 29.986\end{array}$

Table 2. Some frequencies in the fundamental pass band.

\subsection{Bead Pull Measurement}

A standard traveling wave bead pull measurement was performed. In order to keep the perturbation of the fiber small, a very thin pure silk fiber with $0.2 \mathrm{~mm}$ of diameter was used. The bead itself was made by painting the fiber over a length of $0.5 \mathrm{~mm}$ with liquid silver.

The measurements were made in an uncalibrated mode of the NWA. A first measurement, with fiber inside but bead outside the structure, takes the unperturbed reflection $\Gamma_{u p}$. The next run with the bead pulled through the structure measures the perturbed reflection $\Gamma_{p}(\mathrm{z})$. The corrected data $\Gamma_{c o r}$, results from:

$$
\Gamma_{c o r}(\mathrm{z})=\Gamma_{p}(\mathrm{z})-\Gamma_{u p}(\mathrm{z})=c|\mathrm{E}(\mathrm{z})|^{2} e^{-j 2 \Phi(\mathrm{z})} .
$$

The characteristics of the bead, such as material, shape and volume, represented by the constant $\mathrm{c}$ in eq. (2), were not determined. Therefore, no unit is assigned to the electric field in figure 5.

All measurements were made with a bead on the beam trajectory (no transverse off sets). Figures $5-6$ show one result of the non resonant bead pull measurement. The $2 \pi / 3-$ mode has been found at $30.130 \mathrm{GHz}$. The magnitude of the electric field follows from eq. (2) to:

$$
\mathrm{C} *|\mathrm{E}(\mathrm{z})|=\sqrt{\left|\Gamma_{p}(\mathrm{z})-\Gamma_{u p}(\mathrm{z})\right|}=\sqrt{\left|\Gamma_{\text {cor }}(\mathrm{z})\right|}
$$

and is presented in figure 5, where the abscissa indicates the number of mesh points and represents the $\mathrm{z}$ direction. A polar chart of $\Gamma_{c o r}(\mathrm{z})$ with phase information is presented in figure 6 . There one may find a good agreement between the phase advances per cell and the factor 2 in eq. (2). The presented structure is not an infinite periodic structure and terminated with coupling cells. These coupling cells have a decreased volume compared to the inner cavities and an aperture for the power input and output. Therefore the Q-value is different and the resonant frequency is detuned. This mismatch causes an error in the phase advance per cell and in the field magnitude. The attenuation of the modes is also clearly visible.

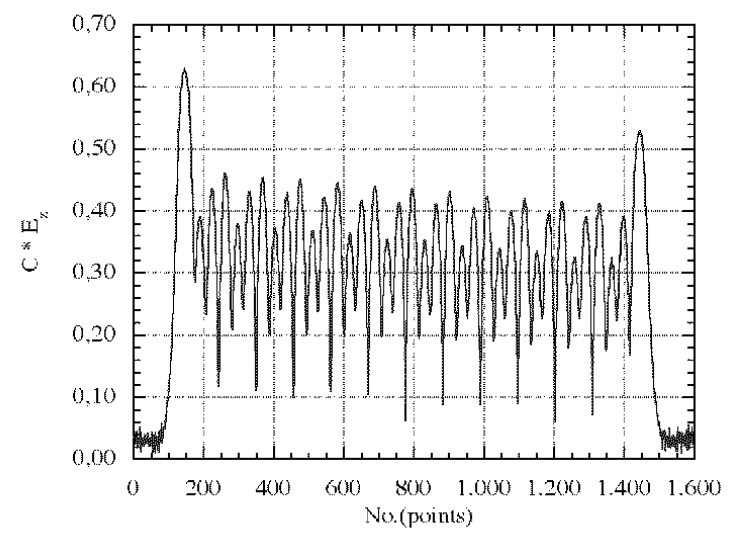

Figure 5. Magnitude of the $2 \pi / 3$-mode at $30.13 \mathrm{GHz}$, determined with bead pull.

90

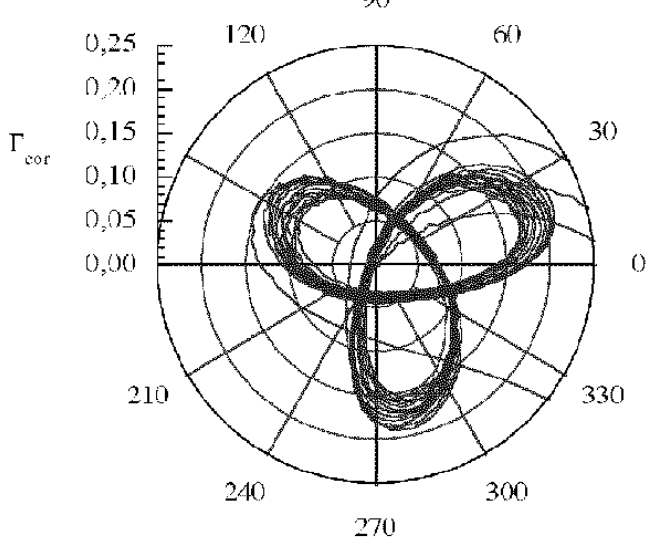

Figure 6. Polar chart of the $2 \pi / 3-$ mode at $30.13 \mathrm{GHz}$, determined with bead pull.

\section{ACKNOWLEDGMENT}

The authors would like to thank the CLIC team for the ideal and material support of the project.

\section{REFERENCES}

[1] W. Bruns, GdfidL: A finite difference program for arbitrarily small perturbations in rectangular geometries, IEEE Trans. Magn. Vol. 32, No. 3 May 1996.

[2] R. Merte, Design of a new R-band 7-cell cold test model RBAND-002, TET Note 98/15, Inst. f. Theoretische Elektrotechnik, TU-Berlin.

[3] R. Merte, Influence of rounded corners seen in X-band RF measurements, TET Note 99/01, Inst. f. Theoretische Elektrotechnik, TU-Berlin.

[4] R. Merte, Realization and RF measurement of a scaled 37cell $X$-band model of the new R-band structure RBAND-003 $E 2$, TET Note 99/02, Inst. f. Theoretische Elektrotechnik, TUBerlin. 